\title{
rhomboid Mediates Specification of Blue- and Green- Sensitive R8 Photoreceptor Cells in Drosophila
}

\author{
Denise A. Birkholz, ${ }^{1}$ Wen-Hai Chou, ${ }^{3}$ Meridee M. Phistry, ${ }^{1}$ and Steven G. Britt ${ }^{1,2}$ \\ ${ }^{1}$ Department of Cell and Developmental Biology and ${ }^{2}$ Department of Ophthalmology and Rocky Mountain Lions Eye Institute, University of Colorado \\ Denver, School of Medicine, Aurora, Colorado 80045, and ${ }^{3}$ Department of Molecular Medicine, University of Texas Health Science Center, San Antonio, \\ Texas 78245
}

Color vision is based on the differential color sensitivity of retinal photoreceptors, however the developmental programs that control photoreceptor cell differentiation and specify color sensitivity are poorly understood. In Drosophila there is growing evidence that the color sensitivity of the R8 cell within an individual ommatidium is regulated by an inductive signal from the adjacent R7 cell. We previously examined the retinal patterning defect in Scutoid mutants, which results from a disruption of rhomboid expression. Here we show that loss of rhomboid blocks the induction of Rh5 expression and misexpression of rhomboid leads to the inappropriate induction of $\mathrm{Rh} 5$. These effects are specific to rhomboid, because its paralogue roughoid is neither required nor sufficient for the induction of $\mathrm{Rh} 5$ expression. We show that rhomboid is required cell-autonomously within the R8 photoreceptor cells and nonautonomously elsewhere in the eye for Rh5 induction. Interestingly, we found that the Epidermal growth factor receptor is also required for Rh5 induction, and its activation is sufficient to rescue the loss of Rh5 induction in a rhomboid mutant. This suggests that rhomboid may function in $\mathrm{R} 8$ cells to activate Epidermal growth factor receptor signaling in R7 cells and promote their differentiation to a signaling competent state.

\section{Introduction}

Drosophila melanogaster is capable of color vision and is a useful experimental system for examining the developmental programs that produce photoreceptor cells having different color sensitivities (Quinn et al., 1974; Spatz et al., 1974; Chou et al., 1996; Chou et al., 1999; Tang and Guo, 2001; Cook et al., 2003; Wernet et al., 2003; Mikeladze-Dvali et al., 2005). The compound eye is composed of $\sim 750$ ommatidia, each containing eight photoreceptor cells (R1-R8) (Hardie, 1985; Heberlein et al., 1993). These cells are distinguishable based on anatomical position, color sensitivity and visual pigment expression (Fig. 1A). The R1-R6 photoreceptor cells express the blue absorbing visual pigment Rhodopsin 1 (Rh1). Adjacent R7 and R8 cells in an individual ommatidium express visual pigments in a precisely coordinated manner (Fig. $1 B-D)$. The two major types of ommatidia are referred to as yellow and pale, based on their appearance under blue illumination (Kirschfeld et al., 1978; Franceschini et al., 1981). Yellow ommatidia occupy $\sim 65 \%$ of the eye and contain UV-sensitive Rh4expressing R7yellow (R7y) cells and green-sensitive Rh6expressing R8yellow (R8y) cells (Fig. 1D). Pale ommatidia

Received Dec. 17, 2008; accepted Jan. 16, 2009.

This work was supported by National Institutes of Health Grant EYR01-12423. We thank Joan Hooper, Sandy Martin, and Lee Niswander for comments on this manuscript, and Matthew Freeman for thoughtful discussion. We thank Lauren Chesnut at the University of Texas Health Science Center at San Antonio for the SEM work.

Correspondence should be addressed to Dr. Steven G. Britt at the above address. E-mail: steve.britt@ucdenver.edu.

W.-H. Chou's present address: Ernest Gallo Clinic and Research Center, University of California, San Francisco, Emeryville, CA 94608.

DOI:10.1523/JNEUROSCI.5988-08.2009

Copyright $\odot 2009$ Society for Neuroscience $\quad$ 0270-6474/09/292666-10\$15.00/0 occupy $\sim 35 \%$ of the eye and contain UV-sensitive Rh3expressing R7pale (R7p) cells and blue-sensitive Rh5expressing R8pale (R8p) cells (Fig. 1C). We have shown that the organization of the R7y and R7p cells is statistically random (Bell et al., 2007), consistent with the idea that R7y versus $\mathrm{R} 7 \mathrm{p}$ cell fate is determined by a stochastic process that requires the gene spineless (Wernet et al., 2006). There is also a specialized class of ommatidia along the dorsal rim (DR) that are thought to be polarization sensitive; DR ommatidia express $\mathrm{Rh} 3$ in both the R7 and R8 cells, which are specified by wingless, the iroquois genes, and homothorax (Fortini and Rubin, 1991; Tomlinson, 2003; Wernet et al., 2003) (Fig. 1B).

The basis for specification of R7 and R8 pairs in yellow and pale ommatidia is unknown, however there is genetic evidence that the coordination of opsin expression in these cells is established by a switch from the default (Rh6, green sensitive) to induced state (Rh5, blue sensitive). This switch within the $\mathrm{R} 8$ cell is dependent on the phenotype of the adjacent R7 cell (Chou et al., 1996, 1999; Papatsenko et al., 1997) (Fig. 1C,D). The presumptive signal from R7 to $\mathrm{R} 8$ is thought to regulate the genes melted and warts that are required for proper Rh5 and Rh6 expression (Mikeladze-Dvali et al., 2005). Here we show that rhomboid ( $r h o$ ) mutants fail to induce the expression of Rh5 in R8 cells adjacent to Rh3-expressing R7 cells. rho is a component of the Epidermal growth factor receptor (EGFR) signaling pathway that is both required and sufficient for the induction of Rh5 expression in R8 cells. These results reveal an unexpected and highly specific role for rho and EGFR in generating photoreceptor cell diversity in the retinal mosaic.

\section{Materials and Methods}

Stocks and genetics. Stocks were maintained in humidified incubators on standard cornmeal/molasses/agar media. Unless otherwise specified, all 
A

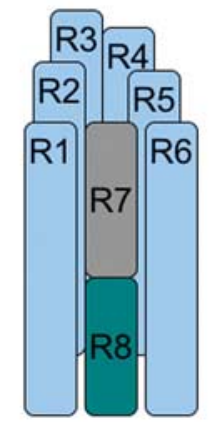

B
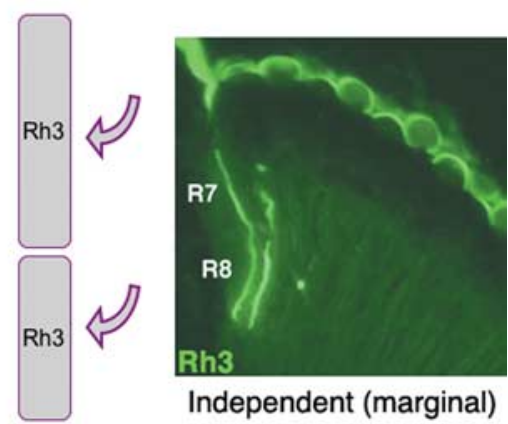

D

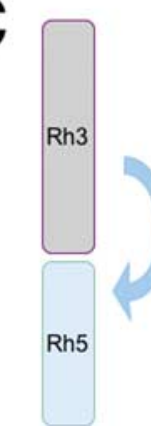

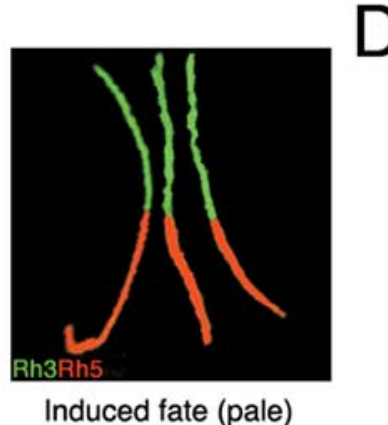

Induced fate (pale)

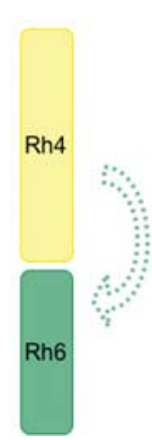

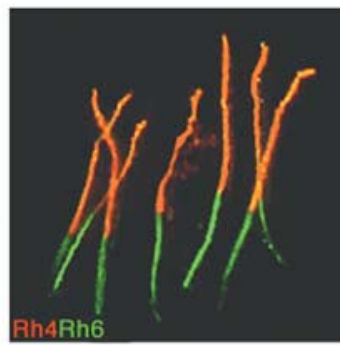

Default fate (yellow)

Figure 1. Patterning of the R7 and R8 photoreceptor cells. $\boldsymbol{A}$, Longitudinal view of a Drosophila ommatidium, composed of eight neuronal photoreceptor cells having rhabdomeres containing the visual pigments. The rhabdomeres of the outer photoreceptor cells (R1-R6) surround the apical and basal rhabdomeres of the inner R7 and R8 cells, respectively. B, Ommatidia along the dorsal margin of the eye contain Rh3 in both R7 and R8. The specification of ommatidia in the dorsal margin occurs from signaling events in the eye periphery. $\boldsymbol{C}, \boldsymbol{D}$, Tangential cross-sections of the two major types of ommatidia, yellow and pale, expressing Rh4 and Rh6 or Rh3 and Rh5 in adjacent R7 and R8 cells. Expression of Rh6 in R8yellow cells $(\boldsymbol{D})$ is not dependent on the presence of an adjacent $R 7$ cell and likely reflects a default fate. Expression of Rh5 in R8pale cells $(\boldsymbol{C})$ is dependent on the presence of an adjacent Rh3-expressing R7 cell and likely reflects an inductive signal.

stocks were obtained from the Bloomington Stock Center. $r h o^{p \Delta 5} F R T$, $r h o^{7 M 43} r u^{l} F R T, r u^{P L L b}$ and UAS-ru were provided by Mathew Freeman. $U A S$-rho was provided by Ethan Bier. UAS-EGFR ${ }^{D N}$ was provided by Nicholas Baker. sina stocks were provided by Richard Carthew. Homozygous mutant clones in the eye of $r h o^{p \Delta 5}$ and $r h o^{7 M 43} r u^{1}$ were generated with the FLP/FRT system by standard techniques (Xu and Rubin, 1993) using the eyeless promoter-driven FLP recombinase (ey-FLP) (Newsome et al., 2000). For ectopic expression, flies containing the UAS-transgenes were crossed to GMR-Gal4 flies at $28^{\circ} \mathrm{C}$. EGFR ${ }^{\text {tsla }} / C y O$ and $E G F R^{f 24} / C y O$ adults were mated and progeny were raised at $18^{\circ} \mathrm{C}$. EGFR $R^{\text {tsla }} / E G F R^{f 24}$ white prepupae $\left(\mathrm{P}_{0}\right)$ were shifted to $29^{\circ} \mathrm{C}$ for $24 \mathrm{~h}$ and returned to $18^{\circ} \mathrm{C}$ until eclosion. As noted in Results, the $E G F R^{E 1} /+$ background yields a significant increase in the percentage of Rh3-expressing R7 cells. We found that this effect is very sensitive to genetic background and have therefore conducted experiments generating $E G F R^{E 1}$, sina, and FLP-FRT rho mutant clones using sibling members of crosses as controls. This is noted in Results and in Table 1.

Scanning electron micrographs. Flies were dehydrated for $12 \mathrm{~h}$ each in a graded ethanol series $(25 \%, 50 \%, 75 \%$ and $100 \%)$, followed by an incubation in hexamethyldisilazane. Samples were dried under house vacuum and sputter coated with a Gold Palladium Target (Electron Microscopy Sciences), mounted on 12-mm-diameter carbon adhesive tabs and placed on stubs. Samples were examined with a LEO 435VP scanning electron microscope (LEO Electron Microscopy Ltd.).

Immunohistochemistry. Cryosections $(10 \mu \mathrm{m})$ were prepared and treated as previously described (Chou et al., 1999). Antibodies were used at the following dilutions: directly conjugated mouse monoclonal antiRh5 (Texas Red, 1:100), anti-Rh6 (FITC, 1:100), anti-Rh3 (clone 2B1, 1:20), anti-Rh4 (clone 11E6, 1:10), FITC-conjugated Fab fragment goat anti-mouse (1:400, Jackson Immunoresearch Laboratories), AlexaFluor 568 goat anti-mouse IgG $(\mathrm{H}+\mathrm{L})(1: 400$, Invitrogen). Immunofluorescence images were acquired with an Axioskop plus/AxioCamHRc (Carl Zeiss) or by confocal microscopy using a Zeiss Pascal LSM (Carl Zeiss).
Comparisons of the proportions (percentages) of opsin expression in different genetic backgrounds were performed with a $z$-score and are shown in Tables 1 and 2 (Fleiss et al., 2003). The $z$-score was calculated using the following equation:

$$
=\frac{\left|p_{2}-p_{1}\right|-\frac{1}{2}\left(1 / n_{1}+1 / n_{2}\right)}{\sqrt{\bar{p} \bar{q}\left(1 / n_{1}+1 / n_{2}\right)}},
$$

where $p_{1}$ and $p_{2}$ are proportions of marker expression in each of the two different genotypes under comparison, $n_{1}$ and $n_{2}$ are the number of ommatidia counted for each genotype, $\bar{p}$ is the average proportion for both genotypes combined, and $\bar{q}=1-\bar{p}$. The significance of the difference between the two proportions was determined from the normal distribution as a two-tailed test.

\section{Results}

rhomboid is both necessary and sufficient for induction of Rh5 expression in R8 photoreceptor cells We previously identified a retinal patterning defect in scutoid mutants that leads to a disruption in the normal paired expression of opsins in adjacent R7 and R8 cells (D. A. Birkholz, W.-H. Chou, M. M. Phistry, and S. G. Britt, unpublished observations). We found that this defect was due to the ectopic expression of the transcriptional repressor snail (Fuse et al., 1999), which causes rho to be misexpressed. To test whether rho regulates specification of R8 photoreceptor cell fate, we generated rho mutant eye patches (Fig. $2 A-D$ ). Interestingly, we observed a dramatic decrease in the number of $\mathrm{R} 8$ cells that expressed $\mathrm{Rh} 5$, accompanied by a corresponding increase in Rh6 expression (Fig. $2 C$; Table 1). Loss of R7 cells, as occurs in the sev mutant, leads to a decrease in Rh5-expressing R8 cells and a corresponding increase in Rh6 (Chou et al., 1999). To verify that a loss of R7 cells did not increase Rh6 expression, we examined a rho mosaic and found that R7 cells are still present and express Rh3 and Rh4 at normal percentages (35\% and $65 \%$ respectively, Table 1$)$. This discrepancy between the normal proportion of Rh3 and Rh4 versus the decrease in Rh 5 suggests that there must be a disruption in the coordination of adjacent R7/R8 cells. Indeed, we found this was the case. rho mutant animals have a large number of ommatidia with mispaired Rh3-expressing R7 cells and Rh6-expressing R8 cells; this effect is specific to the pale class of ommatidia and does not affect the dorsal rim (Fig. 2D). The loss of Rh5 expression in $\mathrm{R} 8$ cells adjacent to Rh3-expressing R7 cells demonstrates that $r$ ho is required for the induction of Rh5 expression, and is essential for establishing R7/R8 cell subtype adjacency (Fig. 1C,D).

To test whether misexpression of rho in WT animals is sufficient for the induction of Rh5 expression and could potentially induce inappropriate induction of Rh5 expression, we used GMR-Gal4 to drive rho expression (UAS-rho) behind the morphogenetic furrow. These animals displayed two mutant phenotypes. First, misexpression of rho roughens the eye (Fig. 2E). As previously shown, ectopic rho expression hyperactivates EGFR signaling and produces excess cone and pigment cells giving the eye a roughened appearance (Wasserman et al., 2000). The second phenotype generated by misexpression of rho is an increase 
Table 1. Opsin expression in different genetic backgrounds

\begin{tabular}{|c|c|c|c|c|}
\hline Genotype & R8 cells expressing Rh5 [\% (n)] & R7 cells expressing Rh3 [\% (n)] & Mispairing & Figure \\
\hline CS & $33 \%(359)$ & $38 \%(139)$ & NO & - \\
\hline$w^{1118}$ & $29 \%(214)$ & $34 \%(140)$ & NO & $1 B-D$ \\
\hline $\operatorname{sev}^{14}$ & $\begin{array}{l}12 \%(585) \\
\operatorname{SDF} C S p<10^{-13}\end{array}$ & - & - & - \\
\hline$r h 0^{p \Delta 5} F R T$ & $\begin{array}{l}6.0 \%(300) \\
\text { SDF } C S p<10^{-15}\end{array}$ & $35 \%(280)$ & $\begin{array}{l}\text { YES } \\
\text { (Rh3/Rh6) }\end{array}$ & $2 A-D$ \\
\hline$r h o^{p \Delta 5} /+$ & $40 \%(208)$ & ND & ND & - \\
\hline$r h o^{7 M 43} r u^{7} F R T$ & No eye & - & - & $3 E$ \\
\hline GMR-GAL4 $\rightarrow$ UAS-EGFR ${ }^{D N}$ & No eye-scar & - & - & $6 C$ \\
\hline$r h o^{7 M 43} r u^{1} /++$ & $40 \%(218)$ & ND & ND & - \\
\hline$r h o^{7 M 43} r u^{7} /+r u^{7}$ & $36 \%(222)$ & ND & ND & $3 D$ \\
\hline$r u^{1}$ & $35 \%(154)$ & $36 \%(191)$ & NO & $3 B$ \\
\hline$r u^{P L L b}$ & $34 \%(339)$ & ND & ND & - \\
\hline GMR-GAL4 $\rightarrow$ UAS-rho & $\begin{array}{l}51 \%(819) \\
\text { SDF CSp }=10^{-8}\end{array}$ & ND & $\begin{array}{l}\text { YES } \\
\text { (Rh4/Rh5) }\end{array}$ & $2 F$ \\
\hline $\begin{array}{l}\text { GMR-rho } \\
y w ; P\{w, G M R-r h o . E x e /\} 3\end{array}$ & $\begin{array}{l}50 \%(250) \\
\operatorname{SDF} C S p<10^{-5}\end{array}$ & $37 \%(590)$ & $\begin{array}{l}\text { YES } \\
\text { (Rh4/Rh5) }\end{array}$ & $6 A$ \\
\hline sev; GMR-rho & $3.4 \%$ (583) SDF GMR-rho $p<10^{-15}$ & ND & ND & $6 B$ \\
\hline GMR-Gal4 $\rightarrow$ UAS-ru & $33 \%$ (231) & ND & NO & $3 F-H$ \\
\hline$E$ RR $^{\text {tsla }} / \mathrm{EGFR}^{\mathrm{CO}}$ & $20 \%(182)$ SDF CS $p=0.0016$ & $37 \%(139)$ & $\begin{array}{l}\text { YES } \\
\text { (Rh3/Rh6) }\end{array}$ & $5 A-E$ \\
\hline$E G F R^{E 1} /+$ & $\begin{array}{l}78 \%(177) \\
\operatorname{SDF}\left(S p<10^{-15}\right.\end{array}$ & $\begin{array}{l}75 \%(236) \\
\text { SDF } C S p<10^{-11}\end{array}$ & $\begin{array}{l}\text { YES } \\
\text { (Rh4/Rh5) }\end{array}$ & $6 D-F$ \\
\hline $\operatorname{sev} ; E_{G F R}{ }^{E 1} /+$ & $\begin{array}{l}32 \%(655) \\
\operatorname{SDF}_{E G F R^{E 1}} p<10^{-15}\end{array}$ & Rh3-expressing R7 cells present & ND & $6 G$ \\
\hline$E G F R^{E 1} /+; \sin a^{2} / s i n a^{3}$ & $\begin{array}{l}15 \%(143) \\
\text { SDF sibling control (below) } p<10^{-13}\end{array}$ & - & - & $6 H$ \\
\hline $\begin{array}{l}\text { sibling control } \\
E_{F F R^{E 1} /+; \sin a^{2} /+}\end{array}$ & $\begin{array}{l}52 \%(443) \\
\operatorname{SDF} C S p<10^{-6}\end{array}$ & $48 \%(96)$ & $\begin{array}{l}\text { YES } \\
\text { Rh3/6, 4/5 }\end{array}$ & - \\
\hline $\sin a^{2} / \sin a^{3}$ & $\begin{array}{l}3.7 \%(404) \\
\text { SDF } C S p<10^{-15}\end{array}$ & - & - & - \\
\hline & $42 \%(258)$ & $51 \%(241)$ & YES & $6 l$ \\
\hline$E G F R^{E 1} /+; r h 0^{p \Delta 5} F R T$ & $\begin{array}{l}\text { Not SDF sibling control (below) } \\
\text { SDF } r h p^{p \Delta 5} F R T p<10^{-15}\end{array}$ & $\begin{array}{l}\text { Not SDF sibling control (below) } \\
\text { SDF CSp }=0.020\end{array}$ & $\operatorname{Rh} 3 / 6,4 / 5$ & \\
\hline $\begin{array}{l}\text { sibling control } \\
E G F R^{E 1} /+; r h 0^{p \Delta 5} /+\end{array}$ & $\begin{array}{l}42 \%(366) \\
\operatorname{SDF} C S p=0.011\end{array}$ & $\begin{array}{l}52 \%(236) \\
\operatorname{SDF} C S p=0.015\end{array}$ & $\begin{array}{l}\text { YES } \\
\text { Rh3/6, 4/5 }\end{array}$ & - \\
\hline
\end{tabular}

Statistical comparisons of strains were carried out as described in Materials and Methods. Parentheses indicate the number of ommatidia counted. Unless indicated, the observed percentages are not significantly different from CS. Strains compared to another control are indicated. SDF, Significantly different from the strain indicated, at the $p$ value shown; ND, not determined; -, not applicable.

in Rh5 expression (51\%, Table 1) and a mispairing of Rh4-expressing R7 cells with Rh5-expressing R8 cells (Fig. 2F). This gain-of-function phenotype is the opposite of the rho loss-of-function phenotype, and demonstrates that rho is sufficient for the induction of Rh5 expression in R8 photoreceptor cells.

\section{roughoid is required for eye} development but not for induction of Rh5 expression in R8 cells

There is a large family of rhomboid-like genes in Drosophila and other organisms (Wasserman et al., 2000; Lee et al., 2001; Freeman, 2008). In addition to $r h o, r u$ is also expressed in the developing Drosophila eye (Wasserman et al., 2000). Previous studies have shown that rho and $r u$ function redundantly in photoreceptor cell recruitment and loss of either gene has a mild phenotype (Figs. 2A, 3A). rho mutants show normal external eye morphology, whereas ru mutants show roughening
Table 2. Opsin expression in rhomboid mosaic ommatidia

\begin{tabular}{|c|c|c|}
\hline Genotype & $\begin{array}{l}\text { R8 cells (adjacent to Rh3-expressing } \\
\text { R7 cells) that express Rh5 [\% (n)] }\end{array}$ & Figure \\
\hline $\begin{array}{l}\mathrm{CS} \\
\mathrm{R} 7^{+} / \mathrm{R}^{+}\end{array}$ & $95 \%(98)$ & - \\
\hline $\begin{array}{l}\text { rho mosaic } \\
\mathrm{R}^{+} / \mathrm{R}^{+}\end{array}$ & $\begin{array}{l}64 \%(120) \\
\operatorname{SDF}\left(S \mathrm{R} 7^{+} / \mathrm{R}^{+}\right. \\
p=1.3 \times 10^{-7}\end{array}$ & $4 A, A^{\prime}$ \\
\hline $\begin{array}{l}\text { rho mosaic } \\
\mathrm{R}^{-} / \mathrm{R}^{+}\end{array}$ & $\begin{array}{l}50 \%(48) \\
\text { Not SDF rho mosaic } \mathrm{R}^{+} / \mathrm{R}^{+}\end{array}$ & $4 B, B^{\prime}$ \\
\hline $\begin{array}{l}\text { rho mosaic } \\
\mathrm{R}^{+} / \mathrm{R}^{-}\end{array}$ & $\begin{array}{l}9 \%(67) \\
\mathrm{SDF} r h o \text { mosaic } \mathrm{R}^{+} / \mathrm{R}^{+} \\
p=9.8 \times 10^{-13}\end{array}$ & $4 C, C^{\prime}$ \\
\hline $\begin{array}{l}\text { rho mosaic } \\
\mathrm{R}^{-} / \mathrm{R}^{-}\end{array}$ & $\begin{array}{l}20 \%(85) \\
\text { SDF rho mosaic R7 }{ }^{+} / \mathrm{R}^{+} \\
p=1.0 \times 10^{-9} \\
\text { Not SDF rho mosaic R7 }{ }^{+} / \mathrm{R}^{-}\end{array}$ & $4 D, D^{\prime}$ \\
\hline
\end{tabular}

Statistical comparisons of strains were carried out as described in Materials and Methods. The genotype of the rhomboid mosaic animals was $y^{d 2} w^{1118}$ P\{ey-FLP.N\}2 P\{GMR-IacZ.C(38.1)\}TPN1 / $W^{*}$; P\{Ubi-GFP.D\}61EF P\{neoFRT\}80B / rho ${ }^{p \Delta 5}$ P\{neoFRT\}80B. The numbers in parentheses indicate the number of ommatidia counted. The observed percentages were compared to the indicated controls and were significantly different from (SDF) or not SDF the strain indicated, at the $p$ value shown. - , Not applicable. 


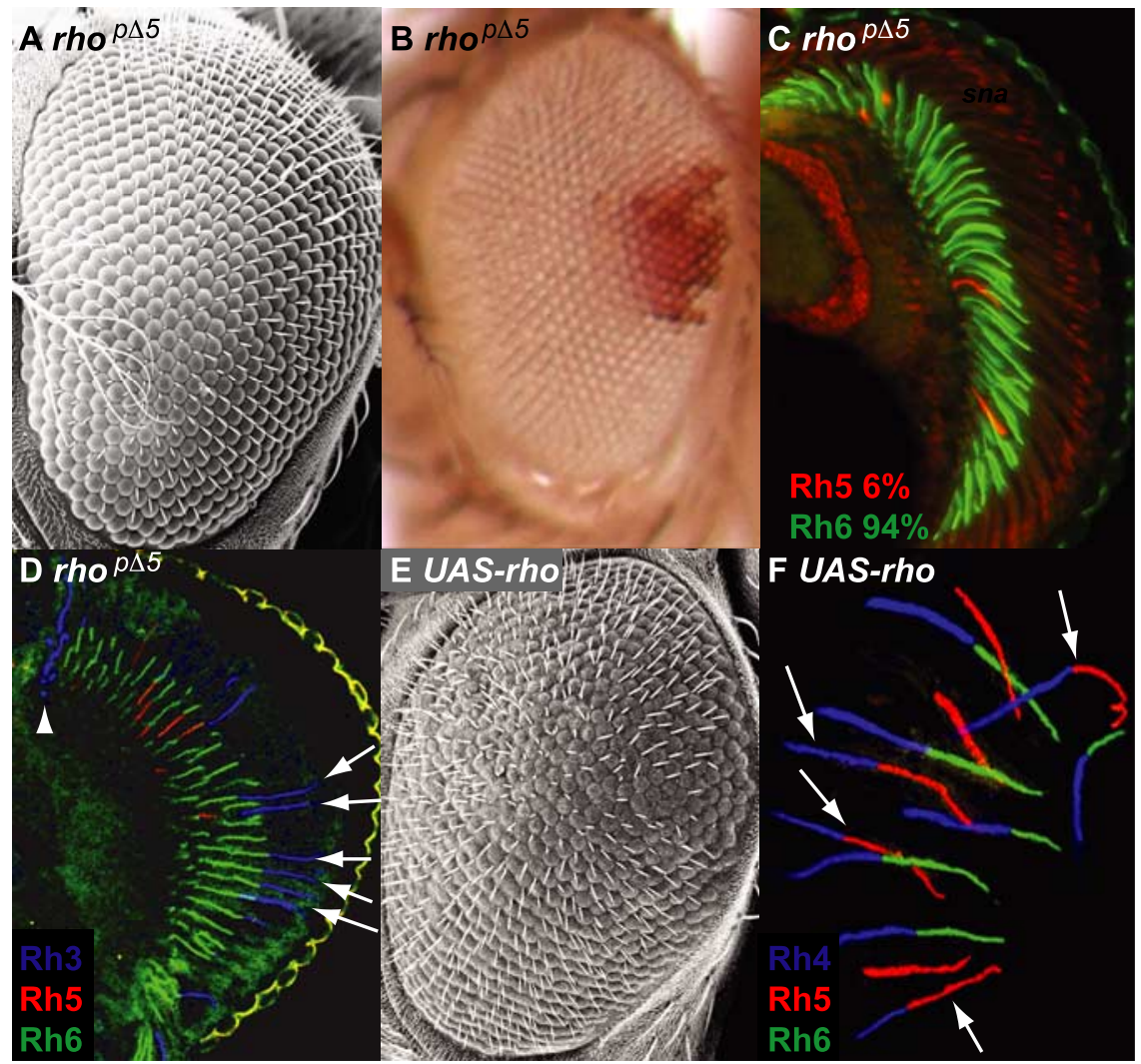

Figure 2. rhomboid is both required and sufficient for induction of Rh5 expression in R8 cells. $\boldsymbol{A}$, Loss of $r$ ho does not dramatically affect the structure of the eye. $\boldsymbol{B}, A$ rho ${ }^{p \Delta 5}$ mosaic eye having a large mutant region (white) and smaller heterozygous region (red). C, A single longitudinal section of the eye in B with an entirely mutant retina labeled with antibodies against Rh5 and Rh6. Removal of rho dramatically increases the number of Rh6-expressing R8 cells compared with $w$. D , Section of an entirely $r$ ho mutant retina labeled with antibodies against $\mathrm{Rh} 3, \mathrm{Rh} 5$, and $\mathrm{Rh} 6$. Loss of Rh5 expression in rho mutants is accompanied by an increase in Rh6 expression and mispairing of $\mathrm{Rh} 3$ and $\mathrm{Rh} 6$ expression in individual ommatidia (arrows), consistent with a loss of induction of Rh5. R7 and R8 cells of the dorsal rim are unaffected and express Rh3 normally (arrowhead). E, Ectopic rho (UAS-rho) driven by GMR-Gal4 results in a rough eye with both missing and double bristles. $\boldsymbol{F}$, Ectopic expression of $r$ o (as in $\boldsymbol{E}$ ) results in an increase in the number of Rh5-expressing R7 cells and a mispairing of Rh4 and Rh5 (arrows).

of the eye and loss of cone cells, pigment cells and some photoreceptor cells (Freeman et al., 1992; Wasserman et al., 2000). However, loss of both genes causes major defects in photoreceptor cell recruitment and eye formation (Fig. $3 E$ ), similar to that observed on removal of EGFR (see Fig. 6C) (Wasserman et al., 2000; Urban et al., 2001). These previous studies showed that the functions of $r h o$ and $r u$ overlap to such an extent that the presence of either gene is sufficient for eye formation, clearly demonstrating that their functions are redundant for eye formation.

To test whether $r u$ may also be required for normal photoreceptor cell patterning, we examined the phenotype of $r u$ loss-offunction mutants. Interestingly, although these animals have the roughened eye previously noted, the normal proportion of Rh5and Rh6-expressing R8 cells are present (Fig. 3B; Table 1), as are Rh3- and Rh4-expressing R7 cells (Table 1). The $r u$ allele shown $\left(r u^{l}\right)$ is a strong loss-of-function allele. We also examined a viable null allele $\left(r u^{P L L b}\right)$ and found the same phenotype (Table 1). Loss of a single copy of $r$ ho in a $r u$ mutant background has no effect on R8 photoreceptor cell differentiation (Fig. 3D; Table 1), whereas animals mutant for rho alone have a very specific defect (Fig. $2 C, D)$. Our studies clearly demonstrate that in the absence of $r h o$, $r u$ alone is not sufficient for R8 photoreceptor cell subtype specification. Furthermore, loss of $r u$ does not sensitize the animal to the dosage of rho that is present. Although rho and ru have redun- dant functions in photoreceptor cell recruitment, there is a specific requirement for $r h o$ and not $r u$ in regulating the expression of Rh5 versus Rh6 in R8 photoreceptor cells.

rho and ru encode structurally related membrane proteases that function in the cleavage and secretion of the EGFR ligands. The requirement for $r$ ho but not $r u$ in $\mathrm{R} 8$ cell differentiation may reflect a different spatial or temporal expression pattern rather than different biochemical functions. To test this hypothesis, we ectopically expressed UAS-ru using the GMR-Gal4 driver described above. If $r u$ has an identical function to rho then expression of $r u$ behind the morphogenetic furrow should be sufficient to induce Rh5 expression in R8 photoreceptor cells. Interestingly, we found that this was not the case. Expression of UAS-ru caused the compound eye to be roughened (Fig. 3F), however opsin gene expression in the R7 and R8 photoreceptor cells was normal (Fig. 3G,H; Table 1). This demonstrates that in contrast to rho, $r u$ is neither required nor sufficient for induction of Rh5 expression in R8 cells.

\section{rhomboid is required in $\mathrm{R} 8$ cells and} elsewhere in the eye for Rh5 induction To determine where rho is normally required for the induction of Rh5 expression, we generated rho mutant clones in a heterozygous background using the FLPFRT system. Cells that are either wild type or heterozygous express GFP, whereas cells that are mutant for rho do not express GFP. We dissociated ommatidia from animals constructed in this manner and counted the expression of Rh5 versus Rh6 in ommatidia in which the genotype of the R7 and R8 cells could be scored. It was essential to conduct this experiment in a rigorous statistical manner, because there are some R8 cells that express Rh5 in a completely rho mutant eye (e.g., Fig. 2C). Furthermore, in previous studies, we have shown that there is occasional mispairing of Rh3 and Rh6 in normal wild-type eyes (signal failure), as well as Rh5 expression in sev animals lacking R7 cells, in which there is no signal to induce Rh5 (Chou et al., 1999).

To compare the effectiveness of Rh5 induction resulting from the R7 to R8 signal, we compared the percentage of Rh5 expression in R8 cells in ommatidia expressing Rh3 in the R7 cell and having readily scoreable $\mathrm{R} 7$ and $\mathrm{R} 8$ cell genotypes. Comparisons of the percentage of opsin expression in ommatidia having R7 and $\mathrm{R} 8$ cells of different genotypes were performed with a $z$-score (Fig. 4; Table 2) (Fleiss et al., 2003). In each panel we show a montage of ommatidia labeled against Rh3, Rh5 and GFP. Wildtype or heterozygous R7 and R8 cells are labeled with GFP, indicated with arrows in each panel, and the GFP image alone is shown as a separate panel (Fig. $4 A^{\prime}$ for example). Cells that are mutant for rho do not express GFP. We found that in ommatidia having genetically mutant R7 cells and genetically wild-type R8 cells (Fig. $4 B, B^{\prime}$ ), the percentage of ommatidia expressing Rh5 


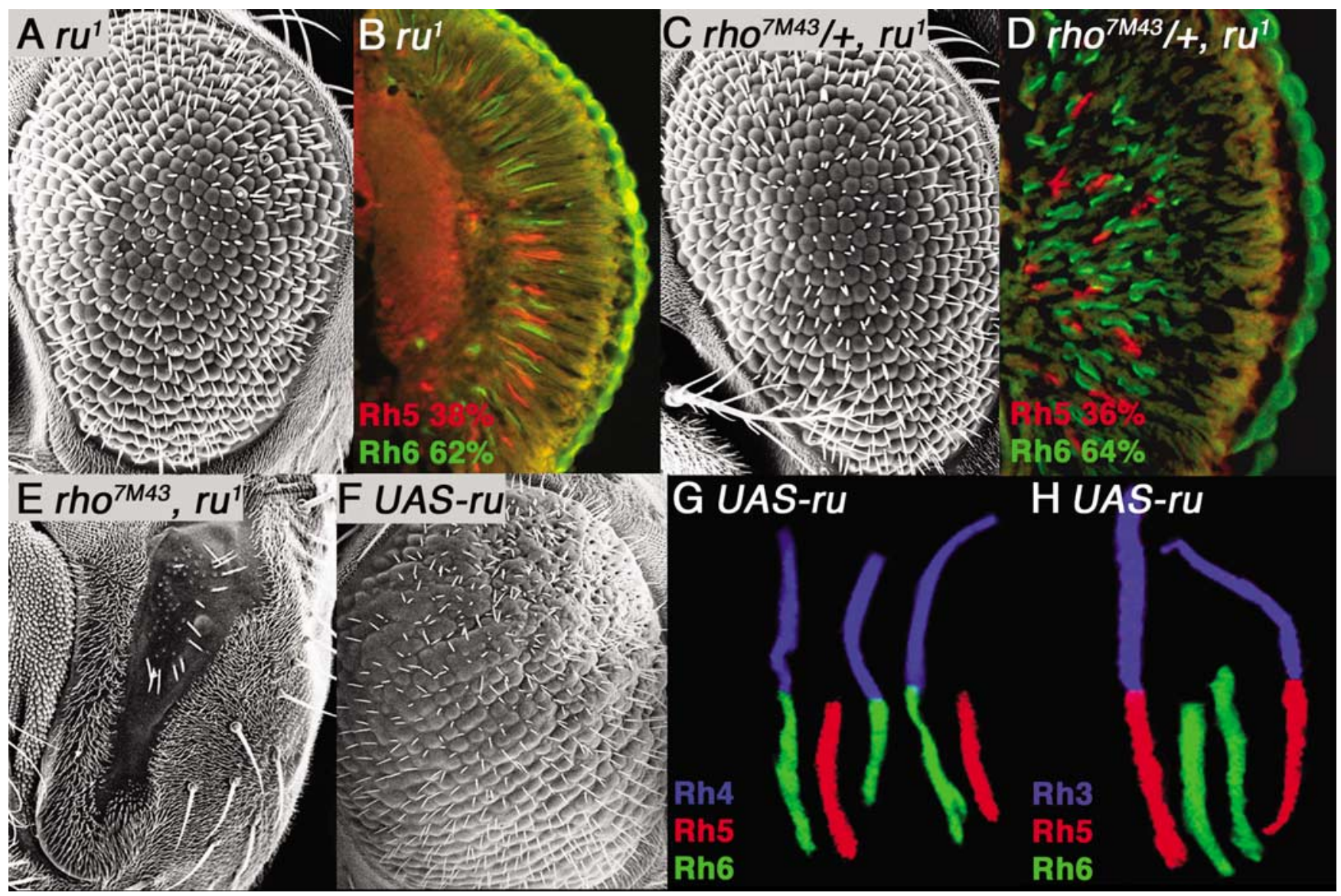

Figure 3. roughoid is neither required nor sufficient for induction of Rh5 expression in R8 cells. $\boldsymbol{A}$, Loss of ru results in a rough eye phenotype. B, Rh5 (35\%) and Rh6 (65\%) expression are normal in $r u^{7}$, a strong hypomorph. We examined a viable null allele, $r u^{P L L b}$, which also has normal Rh5 expression (34\%, Table 1). C, Loss of one copy of rho does not alter the ru rough eye phentoype. $\boldsymbol{D}$, Loss of both copies of $r u$ does not sensitize the eye to the dosage of $r$. Animals heterozygous for $r h o$ and homozygous mutant for $r u\left(r h 0^{7 M 43} r u^{7} /+, r^{7}\right)$ are normal and display WT percentages of Rh5 and Rh6. E, Removal of both $r h o\left(r h 0^{7 M 43}\right.$ ) and $r u\left(r u^{7}\right)$ results in the loss of all ommatidia in the eye, similar to that found with the loss of EGFR function (Fig. 6C). $\boldsymbol{F}$, Expression of ectopic $r u$ (UAS-ru) driven by GMR-Gal4 results in a very rough eye. $G, H$, Ectopic expression of $r u$ does not affect the percentage or pairing of opsin gene expression in the R7 and R8 photoreceptor cells. Normal pairing of Rh4 and Rh6 as well as Rh3 and Rh5 is observed, demonstrating that $r u$ is not sufficient for induction of Rh5 expression in R8 cells (33\%, Table 1).

was not statistically different from ommatidia having genetically wild-type R7 and R8 cells (Fig. $4 A, A^{\prime}$ ) (50\% vs $64 \%$, respectively, Table 2). Conversely, there was a dramatic decrease in Rh5 expression in ommatidia having a genetically mutant $\mathrm{R} 8$ cell and a genetically wild-type $\mathrm{R} 7$ cell (Fig. $\left.4 C, C^{\prime}\right)(9 \%$, Table 2$)$. The percentage of Rh5 expression in ommatidia having both genetically mutant R7 and R8 cells (Fig. 4D, $D^{\prime}, 20 \%$; Table 2), was statistically different from that of ommatidia having genetically wildtype R7 and R8 cells, whereas it was not significantly different from ommatidia having a genetically mutant R8 cell alone (Fig. $\left.4 C, C^{\prime}\right)$. These results demonstrate that signaling is impaired in ommatidia in which the R8 cell is genetically mutant for rho. Impaired signaling is not enhanced when the R7 cell is also genetically mutant. When the R7 cell is genetically mutant and adjacent to a genetically wild-type R8 cell, signaling and induction of $\mathrm{Rh} 5$ expression is the same as when both cells are genetically wild type. One caveat to these results is that we were unable to genotype every cell in the ommatidium. It does appear that there is a nonautonomous requirement for rho elsewhere in the ommatidia or developing eye. We believe this is responsible for the reduction in Rh5 when both R7 and R8 are genetically wild type (64\% vs $95 \%$ for CS, Table 2). These findings demonstrate that $r$ ho function in the R7 cell is not required; however, loss of rho in the R8 cell or elsewhere in the ommatidium or eye compromises induction of $\mathrm{Rh} 5$ expression.
Epidermal growth factor receptor is required for induction of Rh5 expression in R8 photoreceptor cells

rho is an activator of the EGFR pathway throughout most of Drosophila development. EGFR is a transmembrane receptor tyrosine kinase (RTK) that acts reiteratively throughout eye development to regulate cell fate specification, control ommatidial spacing, and inhibit programmed cell death (Freeman, 1996; Domínguez et al., 1998; Kumar et al., 1998). Having demonstrated the requirement for rho in photoreceptor cell subtype specification, we wanted to determine whether EGFR was also required for this process. We used the temperature-sensitive $E G F R$ allele, $E G F R^{t s l a}$, to study the consequences of EGFR loss during eye development. Removal of EGFR for $24 \mathrm{~h}$ during late larval/early pupal development (L3/P0) produced a very rough compound eye (Fig. 5A,B). Loss of EGFR in the anterior eye, before the passage of the morphogenetic furrow, leads to missing ommatidia. Loss of EGFR in the posterior eye, after the passage of the morphogenetic furrow, roughens the eye due to the loss of cone and pigment cells (Kumar et al., 1998). Loss of EGFR also impairs the induction of Rh5 expression and causes an increase in the expression of Rh6 (Fig. 5C; Table 1). Similar to the loss of rho, loss of EGFR does not alter the proportions of Rh3- and $\mathrm{Rh} 4$ expressing R7 cells (Fig. 5D; Table 1), however there is a dramatic mispairing of Rh3-expressing R7 cells with Rh6-expressing R8 cells (Fig. 5E). These findings demonstrate that EGFR is required 
wild type R7 - wild type R8
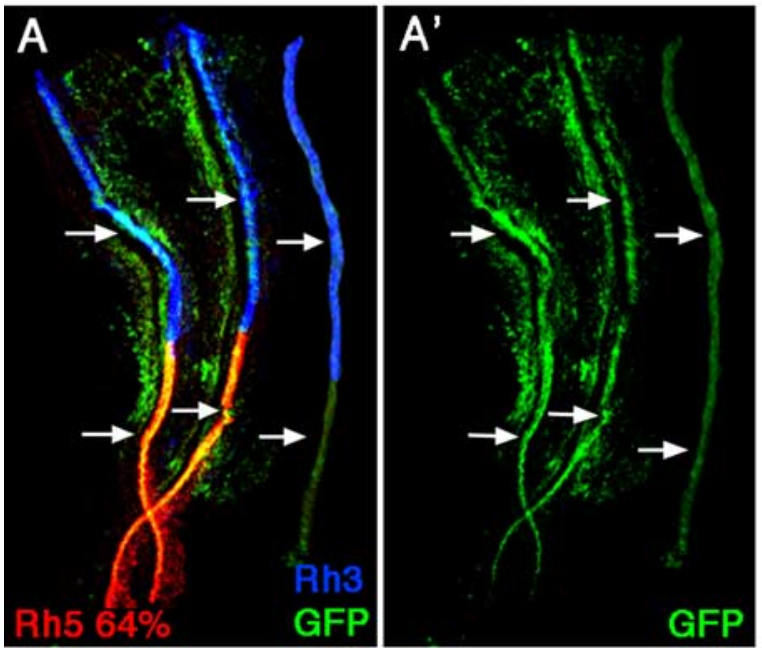

wild type R7 - mutant R8
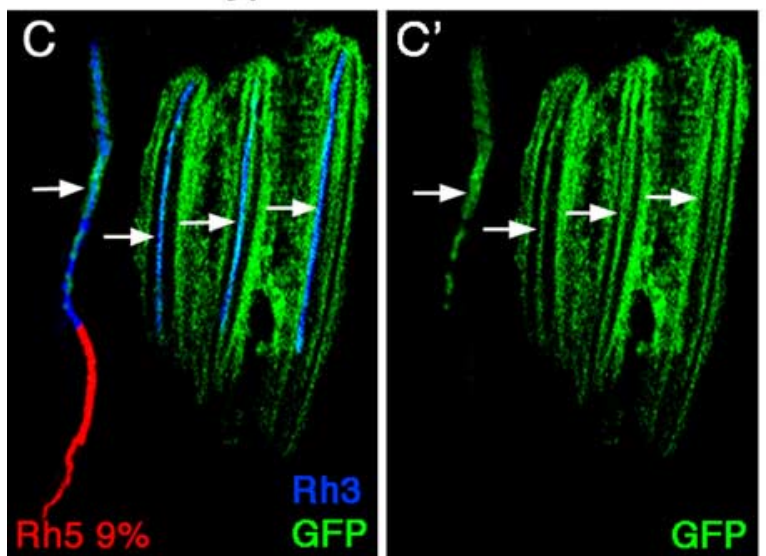

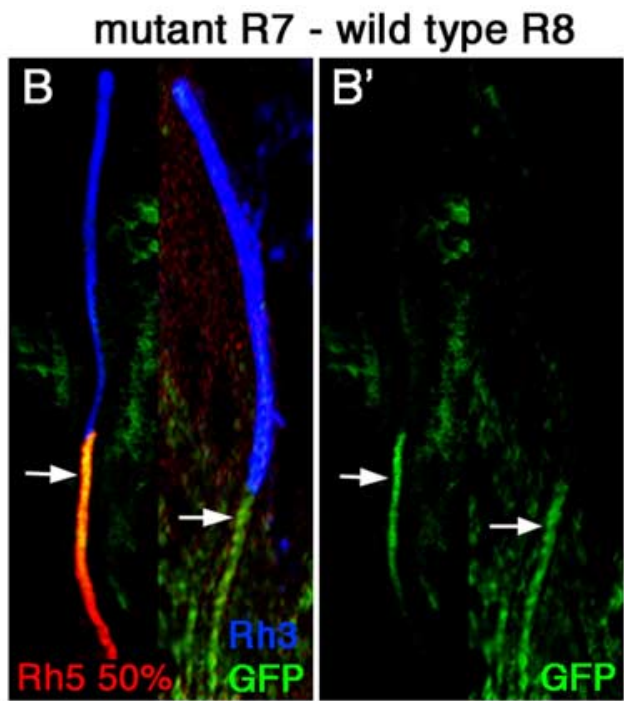

mutant R7 - mutant R8
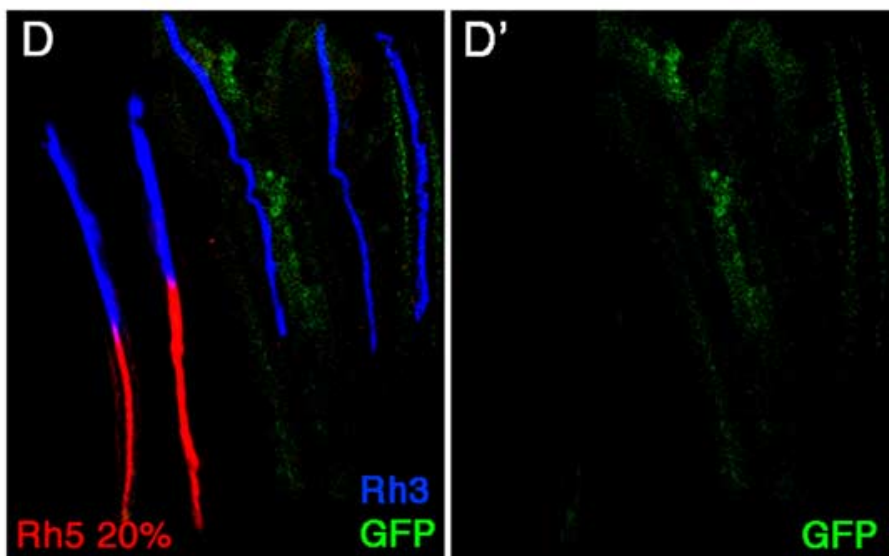

Figure 4. rhomboid is required in R8 photoreceptor cells and elsewhere in the eye for induction of Rh5 expression. rho mutant mosaic animals were constructed having GFP-marked wild-type and rho-heterozygous cells. rho ${ }^{p \Delta 5}$ mutant cells are unlabeled. Each genotype is shown in paired panels, labeled against Rh3, Rh5, and GFP on the left $(\boldsymbol{A}, \boldsymbol{B}, \boldsymbol{C}, \boldsymbol{D})$ and against GFP alone on the right $\left(\boldsymbol{A}^{\prime}\right.$, $\left.\boldsymbol{B}^{\prime}, \boldsymbol{C}^{\prime}, \boldsymbol{D}^{\prime}\right)$. Arrows indicate GFP-marked cells. Statistical comparisons between results are detailed in Table $2 . \boldsymbol{A}, \boldsymbol{A}^{\prime}, 0 \mathrm{mmatidia}$ from $r 0^{p \Delta 5}$ mosaic animals with GFP-marked R7 and R8 cells show $64 \%$ pairing between Rh3- and Rh5-expressing cells. This is significantly less than in completely wild-type (S animals, indicating that $r h o$ is required elsewhere in the eye (see Results and Table 2 ). $B, \boldsymbol{B}^{\prime}, 0$ mmatidia having mutant R7 cells and GFP-marked R8 cells show $50 \%$ pairing between R7 and R8 cells that is not statistically different from the ommatidia in Figure $7 A, A^{\prime}$. $C, \boldsymbol{C}^{\prime}, 0 \mathrm{mmatidia}$ having GFP-marked R7 cells and mutant R8 cells show a dramatic decrease in pairing between R7 and R8 cells ( $9 \%$ ). D, $D^{\prime}, 0$ mmatidia having mutant R7 and R8 cells have reduced pairing (20\%) that is similar to that seen in Figure $7 C, C^{\prime}$.

for regulating the induction of Rh5 expression in R8 cells and establishing R7/R8 photoreceptor cell adjacency.

\section{Epidermal growth factor receptor activation is sufficient to} induce $\mathrm{Rh} 5$ expression and rescue the loss of rhomboid

We found that rho is required and sufficient for the induction of $\mathrm{Rh} 5$ expression. rho is required in the $\mathrm{R} 8$ cell, and the induction of Rh5 expression is also dependent on EGFR. Because of the well described pathway in which EGFR is activated by Spi, after its transport by Star and cleavage by rho, we thought it was likely that a similar process may occur in the regulation of $\mathrm{Rh} 5$ expression and photoreceptor cell patterning. Specifically, we believe that rho may function in the R8 cell to cleave an EGF ligand, that activates EGFR in the R7 cell. Because this signal is directed from the $\mathrm{R} 8$ cell to the $\mathrm{R} 7$ cell, it seems unlikely to be directly responsible for the induction of Rh5 expression. More likely, this rho/ EGFR signal from R8 to R7 may play a role in conferring signaling competency to the R7 cell. This model predicts that (1) induction of Rh5 expression through misexpression of rho is dependent on the presence of the R7 cell, (2) activation of EGFR is sufficient to induce the expression of Rh5, (3) this effect is dependent on the presence of the R7 cell, and (4) activation of EGFR is sufficient to rescue the defect in Rh5 expression found in rho mutant patches.

To test the first prediction, we used a transgene in which GMR directly drives the expression of rho, rather than using the binary GAL4-UAS system. We found that the phenotype of these animals is identical to flies carrying GMR-GAL4; UAS-rho. GMR-rho expressing flies show a dramatic increase in the proportion of Rh5 expressing R8 cells compared with CS (50.4\%, Table 1), a normal proportion of Rh3-expressing R7 cells (37\%, Fig. 6A; Table 1) and mispairing between Rh4-expressing R7 cells and Rh5-expressing R8 cells (Table 1). The GMR-rho transgene was crossed into a sev mutant background that lacks R7 cells, and we found that the increase in Rh5 expression was completely suppressed (Fig. 6B; Table 1), to levels equivalent to that found in animals mutant for sev alone. This finding confirms that induction of Rh5 expression by misexpression of rho is dependent on the presence of the R7 cell and suggests that rho may act on the R7 cell as described in the model above. 

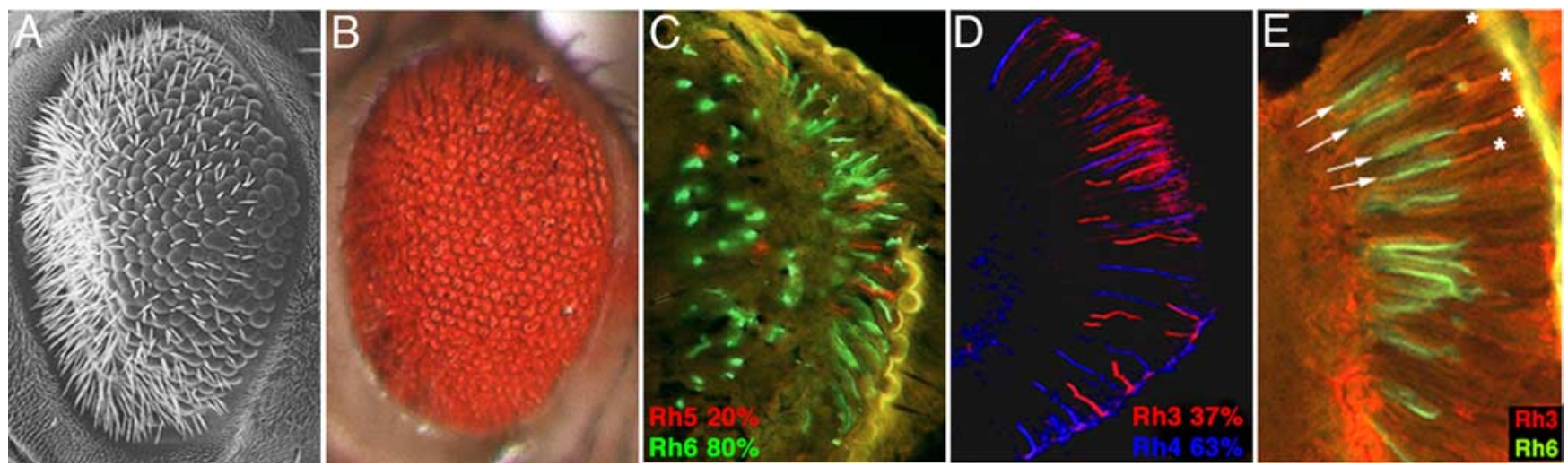

Figure 5. Epidermal growth factor receptor is required for induction of Rh5 expression, and establishment of R7/R8 cell adjacency. $A, S E M$ of an $E G F R^{t s l a} / E G F R^{f 24}$ animal that was heat shocked for $24 \mathrm{~h}$ beginning at $\mathrm{PO}(\sim 240 \mathrm{~h}$ AED). B, Bright-field view of animal treated as in $(\boldsymbol{A})$ shows similar anterior ommatidial loss and roughening throughout. C, Longitudinal section of heat-shocked EGFR animal shown in $\boldsymbol{B}$ displays an increase in Rh6 expression (80\%). D, $\boldsymbol{E}$, In adjacent sections (D) R7 cells are present and expression of Rh3 (37\%) and Rh4 (63\%) is normal, and (E) loss of EGFR results in a mispairing of Rh3-expressing R7 cells with Rh6-expressing R8 cells (white arrows/white asterisks), consistent with a loss of induction of Rh5 expression in R8 cells.

To test the second prediction that activation of EGFR is sufficient to induce Rh5 expression, we examined the phenotype of animals carrying the activated Ellipse allele of EGFR $\left(E G F R^{E 1}\right)$. This mutation causes a single amino acid substitution in the kinase domain of the protein, which activates tyrosine kinase activity. The mutation behaves as a hypermorph, and is effectively a dominant active EGFR (Lesokhin et al., 1999). We found that animals carrying $E G F R^{E 1} /+$ show a dramatic increase in the number of R8 cells that express Rh5 (77\%, Fig. 6D; Table 1); however, this is accompanied by an increase in the number of R7 cells that express Rh3 (75\%, Fig. 6E; Table 1). There are nonetheless instances of mispairing between Rh4-expressing R7 cells and Rh5-expressing R8 cells (Fig. 6F). This finding demonstrates that activation of EGFR is sufficient to induce Rh5 expression, including inappropriate Rh5 expression in R8 cells adjacent to Rh4expressing R7 cells.

To test the third prediction that the increase in Rh5 expression is dependent on the presence of R7 cells, as opposed to the possibility that EGFR activation could be acting on R8 cells to induce Rh5 expression, we examined $E G F R^{E 1}$ in a sev mutant background. In sev; $E G F R^{E 1} /+$ mutants, we found that Rh5 induction was dramatically reduced (32\%) but not eliminated (Fig. 6G; Table 1). Interestingly, some R7 cells remain in this genotype (data not shown) consistent with the ability of EGFR activation to partially rescue loss of the sev receptor tyrosine kinase. To definitively determine whether the $E G F R^{E 1}$ effect on R8 cells is dependent on the presence of R7 cells, we crossed the $E G F R^{E 1}$ allele into seven in absentia (sina), which functions downstream of Ras activation in R7 cell recruitment (Carthew and Rubin, 1990; Fortini et al., 1992). Sibling controls of the genotype $E G F R^{E 1} /+$; sina/+ showed an increase in Rh5-expressing R8 cells compared with $C S$ (51.7\% that was similar to that of $E G F R^{E 1} /+$, Table 1). Furthermore, $E G F R^{E 1}$; sina double mutants that lack R7 cells have a dramatic reduction in the expression of Rh5 (15\%, Fig. 6 H; Table $1)$. These animals are red eyed and demonstrate significant fluorescence background, nonetheless only $3 \mathrm{R} 8$ cells within the montage in Figure $6 \mathrm{H}$ show specific staining for Rh5 (arrowheads). This level of Rh5 expression is comparable although somewhat higher than that found in sina mutants $(3.7 \%$, Table 1 ), and demonstrates that the induction of Rh5 expression on EGFR activation is dependent on the presence of R7 cells.

To test the fourth prediction that activation of EGFR is sufficient to rescue the rho mutant phenotype, we generated flies car- rying the $E G F R^{E 1}$ allele in a background in which $r h o^{p \Delta 5}$ mutant clones were formed. Sibling controls carrying $E G F R^{E 1} /+$, the FRT-FLP components and heterozygous for $r h o^{p \Delta 5}$ show an increased proportion of Rh5-expressing R8 cells compared with CS (42.4\%, Table 1) and numerous examples of Rh3/Rh6, and Rh4/ Rh5 mispaired ommatidia. In $E G F R^{E 1}$ animals having rho ${ }^{p \Delta 5}$ mutant patches, the percentage of Rh5-expressing R8 cells is unchanged (41.9\%, Fig. 6I; Table 1). This demonstrates that in the absence of rho, activation of EGFR is sufficient to induce the expression of Rh5.

\section{Discussion}

The precise pairing of adjacent R7 and R8 photoreceptor cells establishes three very specific classes of ommatidia that have unique sensitivities to different colors and polarized light (Kirschfeld et al., 1978; Hardie, 1979, 1985). We have shown that rho is required to coordinate the differentiation of adjacent R7 and R8 cells over the majority of the retina. Furthermore, we have shown that expression of rho posterior to the morphogenetic furrow is sufficient for the induction of Rh5 expression in R8 cells. Interestingly, although $r h o$ and $r u$ are redundant for early stages of eye formation and retina development, $r u$ is neither required nor sufficient for this process.

\section{Photoreceptor cell subtype specification}

The compound eye of Drosophila is a classic model system in developmental biology, however only recently have the mechanisms that regulate photoreceptor cell subtype specification been investigated. Genes that participate in this process include orthodenticle, prospero, spalt, wingless, the iroquois complex, homothorax, warts, melted, and spineless (Mollereau et al., 2001; Cook et al., 2003; Tahayato et al., 2003; Tomlinson, 2003; Wernet et al., 2003, 2006; Mikeladze-Dvali et al., 2005; Mazzoni et al., 2008). These studies have shown that specific genes are required to (1) activate and repress the transcription of the visual pigment genes, (2) distinguish the R7 and R8 cells from each other, (3) distinguish the R7 cells from each other, (4) distinguish the R7 and R8 cells from the R1-6 cells, (5) distinguish the R7 and R8 cells of the dorsal margin from those of the rest of the retina, and (6) regulate the induced versus default switch in R8. The present study demonstrates the requirement for rho and EGFR in this process and suggests that they may establish the competency of R7 cells to induce Rh5 expression in adjacent R8 photoreceptor cells. 


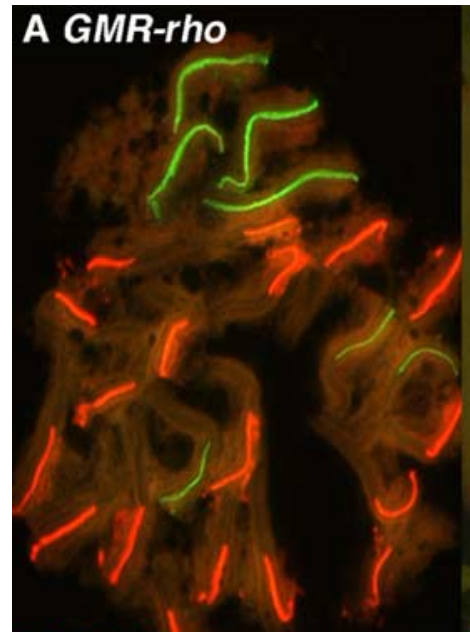

Rh3 37\% Rh 4 63\% D EGFR ${ }^{E 1}$
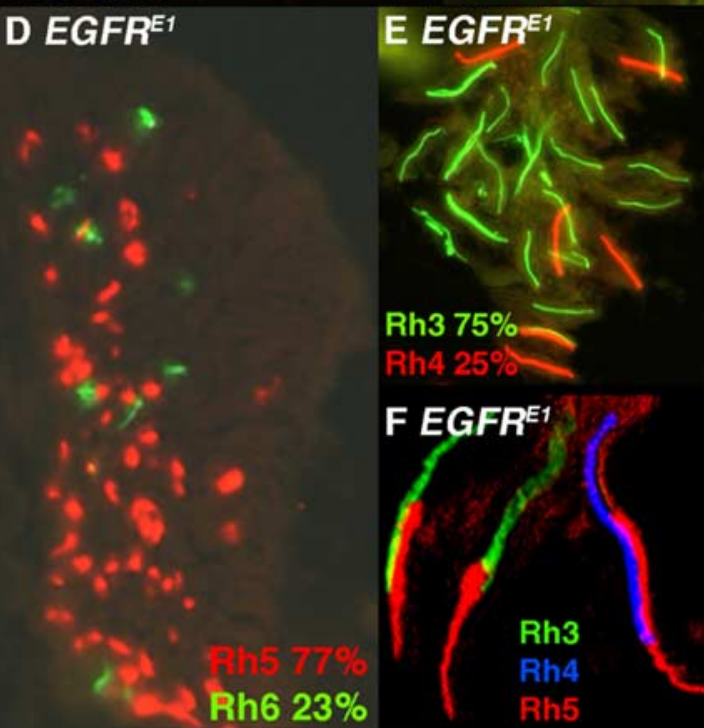

Rh3 $75 \%$
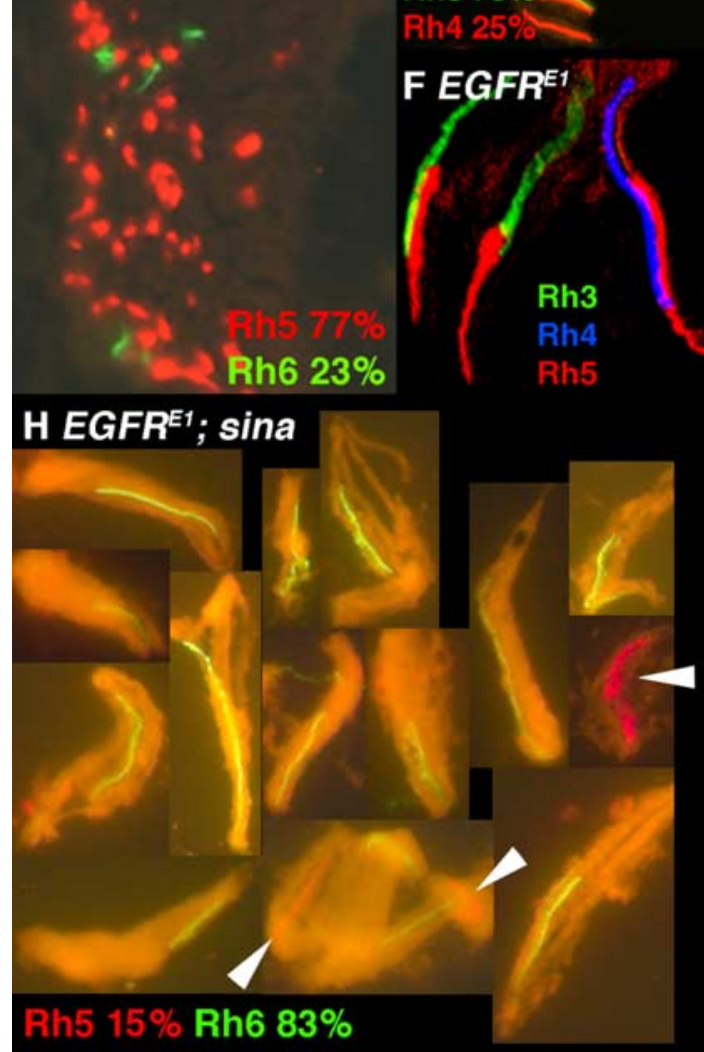

Figure 6. Epidermal growth factor receptor activation is sufficient for induction of Rh5 expression in R8 cells and can rescue the rhomboid mutant phenotype. A, Misexpression of rho under the direct control of GMR does not alter the percentage of Rh3expressing R7 cells (37\%). B Removal of R7 cells in sev; GMR-rho mutant flies results in a dramatic decrease in the percentage of Rh5-expressing R8 cells (3.4\%). C, Downregulation of EGFR throughout the eye with a dominant negative transgene severely reduces the eye similar to the phenotype of rho ru double mutants (Fig. 3E). D, Activation of EGFR in the Ellipse allele leads to a dramatic increase in $\mathrm{Rh} 5$ expression (77\%). $\boldsymbol{E}, \boldsymbol{F}, E G F R^{E 1}$ flies show increased expression of $\mathrm{Rh} 3(75 \%)(\boldsymbol{E})$ and mispairing between Rh4-expressing R7 cells and Rh5-expressing R8 cells $(\boldsymbol{F})$. G, Removal of most R7 cells with the sev mutation suppresses the effect of $E G F R^{E 1}$ on the induction of Rh5 expression (32\%). $\boldsymbol{H}$, Removal of all $\mathrm{R} 7$ cells with the sina mutation leads to further dramatic
In addition to Drosophila, photoreceptor cell subtype adjacency has been observed in the retinal mosaic of teleost fish. The fish retina consists of a crystalline-like array in which rows of red- and greensensitive double cones alternate with rows of UV- and blue-sensitive cones so that each cone type is regularly positioned with respect to other cone types (Stenkamp and Cameron, 2002). It seems likely that one functional consequence of this organization is to ensure adequate spectral sampling throughout the retina, thus preserving high spatial acuity at different wavelengths. Although the basis for photoreceptor cell subtype adjacency in this system is not known, it seems likely that it is established by local inductive signals similar to those we believe regulate this process in Drosophila.

\section{A novel rhomboid function in}

eye development

The recruitment of the R7 photoreceptor cells is a complex process that requires input from at least three signals (Fig. 7). These include contributions from Notch (Cagan and Ready, 1989; Cooper and Bray, 2000; Tomlinson and Struhl, 2001), EGFR (Freeman, 1996; Yang and Baker, 2001), and the boss/sev signaling pathways (Zipursky and Rubin, 1994). Our identification of a requirement for rho in the induction of Rh5 expression in R8 cells, suggests that there is an additional role for EGFR signaling in eye development.

EGFR signaling plays multiple roles in development, both within the eye as well as throughout embryogenesis, imaginal disc differentiation, spermatogenesis, oogenesis and other developmental processes (Shilo, 2003). The principal EGFR ligand in the Drosophila eye is thought to be Spitz (Spi), a TGF- $\alpha$ related, membranetethered molecule that must be activated for complete functioning (Rutledge et al., 1992; Freeman, 1994, 2008; Tio et al., 1994; Schweitzer et al., 1995; Tio and Moses, 1997). Spi first interacts with Star in the endoplasmic reticulum (ER) for transport to the Golgi complex, where it is cleaved by rho and secreted from the signal-sending cell (Freeman, 1994; Bang and Kintner, 2000; Wasserman et al., 2000;

$\leftarrow$

suppression in the effect (Rh5 expression, 15\%). Arrowheads indicate the few remaining Rh5-expressing R8 cells. I, In contrast, loss of rho does not significantly suppress the $E G F R^{E 1}$ induction of Rh5 expression (42\%), compared with sibling controls $\left(E G F R^{E 1} /+; r h o /+, 42 \%\right)$. This demonstrates that loss of Rh5 expression in rho mutant patches can be rescued by EGFR activation. 


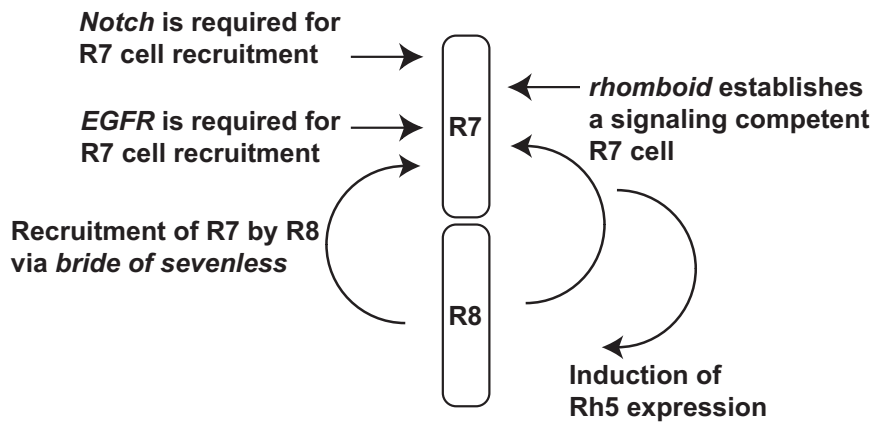

Figure 7. Model for signaling events regulating recruitment and patterning of R7 and R8 photoreceptor cells. There are three distinct requirements for Notch, the Epidermal growth factor receptor, and bride of sevenless/sevenless signaling in the recruitment and induction of R7 cells (left side). The current work demonstrates a specific requirement for rhomboid function in R8 cells and elsewhere in the eye that may confer signaling competency to R7 cells, and allow induction of Rh5 expression and the establishment of precise cell- cell adjacency (right side).

Lee et al., 2001; Urban et al., 2001; Tsruya et al., 2002). rho has also been shown to cleave Star and may thus participate in a negative feedback loop to restrict the amount of Spi that is trafficked from the ER to the Golgi (Tsruya et al., 2007). It is currently thought that three waves of spi/EGFR signaling occur during eye development. In the first wave, rho expression in R8, R5 and R2 cells leads to Spi activation and recruitment of the remaining photoreceptors (R1, R3, R4, R6 and R7). These cells then initiate a second wave of signaling to recruit the cone cells, which then initiate a third wave to recruit the pigment cells (Freeman, 1996). There is additional evidence that $E G F R$ is required in the developing eye for cell proliferation and survival (Domínguez et al., 1998).

These and other recent studies indicate that both the R7 and R8 cells express rho during the period when the cells are recruited and specified, and when Rh5 expression is likely to be induced (Yogev et al., 2008). The precise time at which Rh5 expression is induced in R8 cells is unknown, however it must follow the recruitment and specification of the R7 cells during late third instar and early pupal development, and precede the expression of the opsin genes beginning at $79 \%$ of pupal development (Earl and Britt, 2006).

Because rho is required in the R8 cell (and elsewhere in the eye) and not in the R7 cell, it is unlikely that a rho/EGFR mediated signal is directly responsible for the induction of $\mathrm{Rh} 5$ expression in R8 cells. Nonetheless, our observation that induction of Rh5 expression by GMR-rho misexpression is blocked in sev mutants suggests that $r$ o is sufficient to induce signaling inappropriately in Rh4-expressing cells, but that this is dependent on the presence of the R7 cell. Furthermore, our observation that activation of EGFR can rescue the rho mutant phenotype suggests that rho functions upstream of EGFR activation in the induction of Rh5 expression. This suggests that rho and EGFR may function to establish a signaling competent R7 cell that can then induce Rh5 expression in the R8 cell (Fig. 7). This rho function could be a component of the requirement for EGFR signaling in R7 cell recruitment mentioned above, or could be an additional rhospecific requirement that regulates R7 cell signaling competency. Given that loss of rho or EGFR does not affect the proportion of Rh3- and Rh4-expressing R7 cells, our results suggest that they may act after or independently of spineless (Wernet et al., 2006).

In the context of rho specificity, rho and $r u$ belong to a conserved family of rhomboid proteins that are intracellular membrane proteases (Wasserman et al., 2000; Lee et al., 2001; Urban et al., 2002). The similarity of their enzymatic functions in vitro and their genetic redundancy has led to the proposal that they have limited specificity with regard to substrate cleavage and function within the EGFR signaling pathway (Wasserman et al., 2000; Lee et al., 2001; Urban et al., 2002). Obviously, the fact that $r u$ is not required for regulating R8 cell color sensitivity may simply reflect that it is not expressed at the appropriate location or time. Similarly, it is possible that the inability of misexpressed $r u$ to induce Rh5 expression may result from inadequate expression under the GAL4/UAS system. However, the observation that $r u$ is not sufficient for induction of $\mathrm{Rh} 5$ expression suggests that despite the similar biochemical activity of rho and ru in vitro, their functions in vivo are quite specific. This raises the intriguing possibility that rho and ru may act on different substrates or ligands, require different cofactors, or they may generate ligands that are processed or modulated differently, producing qualitatively different signals. In addition, it was recently shown that rho may function distinctly from $r u$, based on differences in intracellular localization, cleavage of the chaperone Star, and the temporal and quantitative regulation of Spitz trafficking (Yogev et al., 2008). Each of these raises potential mechanisms by which $r h o$ and $r u$ may function in a distinct manner and may underly the specific requirement for $r h o$ and not $r u$ in the induction of Rh5 expression.

Thus in addition to demonstrating the requirement for rho and EGFR in the induction of Rh5 expression, our work raises many questions concerning their mechanism of action. Perhaps the two most important issues to be resolved in future studies are (1) what are the functions of the traditional components of EGFR signaling, Spi and Star, in the induction of Rh5 expression, and (2) what are the temporal and spatial requirements for rho and $E G F R$ with respect to the functions of genes known to establish R7 and R8 photoreceptor cell subtype identity (spineless, warts, and melted)? Answering these questions will help place the function of rho and EGFR in R8 cell subtype specification into context with the activities of other genes and EGFR signaling processes more broadly and better define the role of inductive signaling in retinal development overall.

\section{References}

Bang AG, Kintner C (2000) Rhomboid and Star facilitate presentation and processing of the Drosophila TGF-alpha homolog Spitz. Genes Dev 14:177-186.

Bell ML, Earl JB, Britt SG (2007) Two types of Drosophila R7 photoreceptor cells are arranged randomly: a model for stochastic cell-fate determination. J Comp Neurol 502:75-85.

Cagan RL, Ready DF (1989) Notch is required for successive cell decisions in the developing Drosophila retina. Genes Dev 3:1099-1112.

Carthew RW, Rubin GM (1990) seven in absentia, a gene required for specification of R7 cell fate in the Drosophila eye. Cell 63:561-577.

Chou WH, Hall KJ, Wilson DB, Wideman CL, Townson SM, Chadwell LV, Britt SG (1996) Identification of a novel Drosophila opsin reveals specific patterning of the R7 and R8 photoreceptor cells. Neuron 17:1101-1115.

Chou WH, Huber A, Bentrop J, Schulz S, Schwab K, Chadwell LV, Paulsen R, Britt SG (1999) Patterning of the R7 and R8 photoreceptor cells of Drosophila: evidence for induced and default cell-fate specification. Development 126:607-616.

Cook T, Pichaud F, Sonneville R, Papatsenko D, Desplan C (2003) Distinction between color photoreceptor cell fates is controlled by Prospero in Drosophila. Dev Cell 4:853-864.

Cooper MT, Bray SJ (2000) R7 photoreceptor specification requires Notch activity. Curr Biol 10:1507-1510.

Domínguez M, Wasserman JD, Freeman M (1998) Multiple functions of the EGF receptor in Drosophila eye development. Curr Biol 8:1039-1048.

Earl JB, Britt SG (2006) Expression of Drosophila rhodopsins during photoreceptor cell differentiation: insights into R7 and R8 cell subtype commitment. Gene Expr Patterns 6:687-694. 
Fleiss JL, Levin BA, Paik MC (2003) Statistical methods for rates and proportions, Ed 3. Hoboken, NJ: Wiley-Interscience.

Fortini ME, Rubin GM (1991) The optic lobe projection pattern of polarization-sensitive photoreceptor cells in Drosophila melanogaster. Cell Tissue Res 265:185-191.

Fortini ME, Simon MA, Rubin GM (1992) Signalling by the sevenless protein tyrosine kinase is mimicked by Ras1 activation. Nature 355:559-561.

Franceschini N, Kirschfeld K, Minke B (1981) Fluorescence of photoreceptor cells observed in vivo. Science 213:1264-1267.

Freeman M (1994) The spitz gene is required for photoreceptor determination in the Drosophila eye where it interacts with the EGF receptor. Mech Dev 48:25-33.

Freeman M (1996) Reiterative use of the EGF receptor triggers differentiation of all cell types in the Drosophila eye. Cell 87:651-660.

Freeman M (2008) Rhomboid proteases and their biological functions. Annu Rev Genet 42:191-210.

Freeman M, Kimmel BE, Rubin GM (1992) Identifying targets of the rough homeobox gene of Drosophila: evidence that rhomboid functions in eye development. Development 116:335-346.

Fuse N, Matakatsu H, Taniguchi M, Hayashi S (1999) Snail-type zinc finger proteins prevent neurogenesis in Scutoid and transgenic animals of Drosophila. Dev Genes Evol 209:573-580.

Hardie RC (1979) Electrophysiological analysis of fly retina. I: comparative properties of R 1-6 and R 7 and 8. J Comp Physiol 129:19-33.

Hardie RC (1985) Functional organization of the fly retina. In: Progress in sensory physiology (Autrum H, Ottoson D, Perl ER, Schmidt RF, Shimazu H, Willis WD, eds), pp 1-79. Berlin: Springer.

Heberlein U, Wolff T, Rubin GM (1993) The TGF beta homolog dpp and the segment polarity gene hedgehog are required for propagation of a morphogenetic wave in the Drosophila retina. Cell 75:913-926.

Kirschfeld K, Feiler R, Franceschini N (1978) A photostable pigment within the rhabdomeres of fly photoreceptors no. 7. J Comp Physiol 125:275-284.

Kumar JP, Tio M, Hsiung F, Akopyan S, Gabay L, Seger R, Shilo BZ, Moses K (1998) Dissecting the roles of the Drosophila EGF receptor in eye development and MAP kinase activation. Development 125:3875-3885.

Lee JR, Urban S, Garvey CF, Freeman M (2001) Regulated intracellular ligand transport and proteolysis control EGF signal activation in Drosophila. Cell 107:161-171.

Lesokhin AM, Yu SY, Katz J, Baker NE (1999) Several levels of EGF receptor signaling during photoreceptor specification in wild-type, Ellipse, and null mutant Drosophila. Dev Biol 205:129-144.

Mazzoni EO, Celik A, Wernet MF, Vasiliauskas D, Johnston RJ, Cook TA, Pichaud F, Desplan C (2008) Iroquois complex genes induce coexpression of rhodopsins in Drosophila. PLoS Biol 6:e97.

Mikeladze-Dvali T, Wernet MF, Pistillo D, Mazzoni EO, Teleman AA, Chen YW, Cohen S, Desplan C (2005) The growth regulators warts/lats and melted interact in a bistable loop to specify opposite fates in Drosophila R8 photoreceptors. Cell 122:775-787.

Mollereau B, Dominguez M, Webel R, Colley NJ, Keung B, de Celis JF, Desplan C (2001) Two-step process for photoreceptor formation in Drosophila. Nature 412:911-913.

Newsome TP, Asling B, Dickson BJ (2000) Analysis of Drosophila photoreceptor axon guidance in eye-specific mosaics. Development 127:851-860.

Papatsenko D, Sheng G, Desplan C (1997) A new rhodopsin in R8 photoreceptors of Drosophila: evidence for coordinate expression with Rh3 in R7 cells. Development 124:1665-1673.

Quinn WG, Harris WA, Benzer S (1974) Conditioned behavior in Drosophila melanogaster. Proc Natl Acad Sci U S A 71:708-712.
Rutledge BJ, Zhang K, Bier E, Jan YN, Perrimon N (1992) The Drosophila spitz gene encodes a putative EGF-like growth factor involved in dorsalventral axis formation and neurogenesis. Genes Dev 6:1503-1517.

Schweitzer R, Shaharabany M, Seger R, Shilo BZ (1995) Secreted Spitz triggers the DER signaling pathway and is a limiting component in embryonic ventral ectoderm determination. Genes Dev 9:1518-1529.

Shilo BZ (2003) Signaling by the Drosophila epidermal growth factor receptor pathway during development. Exp Cell Res 284:140-149.

Spatz HC, Emanns A, Reichert H (1974) Associative learning of Drosophila melanogaster. Nature 248:359-361.

Stenkamp DL, Cameron DA (2002) Cellular pattern formation in the retina: retinal regeneration as a model system. Mol Vis 8:280-293.

Tahayato A, Sonneville R, Pichaud F, Wernet MF, Papatsenko D, Beaufils P, Cook T, Desplan C (2003) Otd/Crx, a dual regulator for the specification of ommatidia subtypes in the Drosophila retina. Dev Cell 5:391-402.

Tang S, Guo A (2001) Choice behavior of Drosophila facing contradictory visual cues. Science 294:1543-1547.

Tio M, Moses K (1997) The Drosophila TGF alpha homolog Spitz acts in photoreceptor recruitment in the developing retina. Development 124:343-351.

Tio M, Ma C, Moses K (1994) spitz, a Drosophila homolog of transforming growth factor-alpha, is required in the founding photoreceptor cells of the compound eye facets. Mech Dev 48:13-23.

Tomlinson A (2003) Patterning the peripheral retina of the fly: decoding a gradient. Dev Cell 5:799-809.

Tomlinson A, Struhl G (2001) Delta/Notch and Boss/Sevenless signals act combinatorially to specify the Drosophila R7 photoreceptor. Mol Cell 7:487-495.

Tsruya R, Schlesinger A, Reich A, Gabay L, Sapir A, Shilo BZ (2002) Intracellular trafficking by Star regulates cleavage of the Drosophila EGF receptor ligand Spitz. Genes Dev 16:222-234.

Tsruya R, Wojtalla A, Carmon S, Yogev S, Reich A, Bibi E, Merdes G, Schejter E, Shilo BZ (2007) Rhomboid cleaves Star to regulate the levels of secreted Spitz. EMBO J 26:1211-1220.

Urban S, Lee JR, Freeman M (2001) Drosophila rhomboid-1 defines a family of putative intramembrane serine proteases. Cell 107:173-182.

Urban S, Lee JR, Freeman M (2002) A family of Rhomboid intramembrane proteases activates all Drosophila membrane-tethered EGF ligands. EMBO J 21:4277-4286.

Wasserman JD, Urban S, Freeman M (2000) A family of rhomboid-like genes: Drosophila rhomboid-1 and roughoid/rhomboid-3 cooperate to activate EGF receptor signaling. Genes Dev 14:1651-1663.

Wernet MF, Labhart T, Baumann F, Mazzoni EO, Pichaud F, Desplan C (2003) Homothorax switches function of Drosophila photoreceptors from color to polarized light sensors. Cell 115:267-279.

Wernet MF, Mazzoni EO, Celik A, Duncan DM, Duncan I, Desplan C (2006) Stochastic spineless expression creates the retinal mosaic for colour vision. Nature 440:174-180.

Xu T, Rubin GM (1993) Analysis of genetic mosaics in developing and adult Drosophila tissues. Development 117:1223-1237.

Yang L, Baker NE (2001) Role of the EGFR/Ras/Raf pathway in specification of photoreceptor cells in the Drosophila retina. Development 128:1183-1191.

Yogev S, Schejter ED, Shilo BZ (2008) Drosophila EGFR signalling is modulated by differential compartmentalization of Rhomboid intramembrane proteases. EMBO J 27:1219-1230.

Zipursky SL, Rubin GM (1994) Determination of neuronal cell fate: lessons from the R7 neuron of Drosophila. Annu Rev Neurosci 17:373-397. 